\title{
Overt leukemic phase: an unusual presentation of angioimmunoblastic lymphoma
}

\author{
Komal Arora $^{1} \cdot$ Lingxin Zhang ${ }^{2} \cdot$ Wei Xie $^{3} \cdot$ Kelty R. Baker $^{4} \cdot$ Youli Zu $^{5} \cdot$ Suyang Hao $^{5}$
}

Received: 25 February 2016 / Accepted: 4 July 2016 / Published online: 29 July 2016

(C) Springer-Verlag Berlin Heidelberg 2016

\begin{abstract}
Peripheral blood dissemination has been described in various types of B- and T-cell non-Hodgkin lymphomas. The likelihood of leukemic dissemination differs according to the type of lymphoma. In some cases, a leukemic phase may be the only presentation of the underlying lymphoma and hence careful examination of the peripheral blood smear may suggest a clue to the underlying disease process. However, leukemic dissemination is usually not a feature of angioimmunoblastic T-cell lymphoma (AITL). We describe a case of a 64-year-old woman who presented to our hospital with overt peripheral blood involvement by AITL.
\end{abstract}

Keywords Angioimmunoblastic lymphoma $\cdot$ Leukemic phase $\cdot \mathrm{CD} 10+\mathrm{T}$ cells

Komal Arora

karora@mdanderson.org; arorkomal@gmail.com

Suyang Hao

shao@houstonmethodist.org

1 Department of Pathology and Laboratory Medicine, The University of Texas MD Anderson Cancer Center, Unit 0800, 1515 Holcombe Blvd, Houston, TX 77030, USA

2 Department of Pathology and Immunology, Washington University School of Medicine, St. Louis, MO, USA

3 Department of Hematology and Internal Medicine, Houston Methodist Hospital, Houston, TX, USA

4 Department of Pathology and Immunology, Baylor College of Medicine, Houston, TX, USA

5 Department of Pathology and Genomic Medicine, Houston Methodist Hospital, 6565 Fannin St, Houston, TX 77030, USA

\section{Introduction}

Angioimmunoblastic T-cell lymphoma (AITL) is a distinct clinicopathological entity among peripheral T-cell lymphomas. Patients usually present with generalized lymphadenopathy, hepatosplenomegaly, skin rash, bone marrow involvement, and constitutional symptoms. Notably, hematologic abnormalities such as Coombs-positive hemolytic anemia and polyclonal hypergammaglobulinemia are also present in these patients. AITL was initially thought to be an immunological reactive disorder with high risk of transformation to malignant lymphoma and hence was termed angioimmunoblastic lymphadenopathy $[1,2]$. Later, the discovery of clonal rearrangements of T-cell receptor genes in the majority of cases led to its classification as a T-cell lymphoma. Although a few studies showed that small numbers of AITL cells can be identified in the peripheral blood, an overt leukemic phase has not been known in this disease. We report here the case of a 64year-old woman who presented with fever, leukocytosis, and lymphadenopathy and was found to have a large number of circulating lymphoma cells with an immunophenotypic profile identical to the malignant cells seen in the affected lymph nodes.

\section{Clinical history}

A 64-year-old woman with a 7-year history of dermatomyositis presented at another hospital with a 2-month history of fever, night sweats, and weight loss associated with fatigue, diffuse pruritus, and resultant petechial excoriations of her skin. She had previously undergone treatment with mycophenolate mofetil, methotrexate, and intravenous immunoglobulin for the dermatomyositis; at the time of presentation, however, she was taking only prednisone. Her physical examination was 
remarkable for tachycardia, a cushingoid face, skin and conjunctival pallor, mucosal dryness, axillary and inguinal lymphadenopathy measuring $2-3 \mathrm{~cm}$ each, and hepatosplenomegaly with a total liver span of $16 \mathrm{~cm}$ and spleen extending $8 \mathrm{~cm}$ below the left costal margin.

The laboratory workup at admission revealed a hemoglobin level of $8.2 \mathrm{~g} / \mathrm{dL}$, platelet count of $13 \times 10^{9} / \mathrm{L}$, and white cell count $17.5 \times 10^{9} / \mathrm{L}$ with absolute lymphocytosis. The differential count showed $43 \%$ neutrophils, $53 \%$ atypical lymphocytes, $2 \%$ eosinophils, $1 \%$ monocytes, and $1 \%$ metamyelocytes. Other laboratory findings included a creatinine level of $1.6 \mathrm{mg} / \mathrm{dL}$ (normal $0.5-1.5$ ), an elevated alkaline phosphatase of $148 \mathrm{IU} / \mathrm{L}$ (normal 30-115), elevated erythrocyte sedimentation rate of $38 \mathrm{~mm} / \mathrm{h}$, and a markedly elevated lactate dehydrogenase level of $1051 \mathrm{U} / \mathrm{L}$ (normal 300-600). Excisional biopsy of the inguinal lymph nodes and a staging bone marrow biopsy and aspiration were performed at the outside hospital and slides were sent to the Houston Methodist Hospital (HMH) for interpretation.

\section{Materials and methods}

Hematoxylin and eosin (H\&E)-stained sections of the lymph node biopsy specimen and bone marrow biopsy specimen performed at the outside institution were reviewed at $\mathrm{HMH}$. Immunohistochemical stains were performed on 5- $\mu \mathrm{m}$, formalin-fixed, paraffin-embedded (FFPE) tissue sections from of both specimens using prediluted antibodies (Dako, Carpinteria, CA). Appropriate negative and positive controls were used for each immunostain.

Wright stain was performed on the peripheral blood. Specimens of the peripheral blood and a repeat staging bone marrow biopsy and aspiration done at $\mathrm{HMH}$ were subjected to eight-color flow cytometric evaluation. The resulting data were analyzed by the FACsDiva software (BD Biosciences, San Jose, CA) using a population-based gating strategy. FFPE sections were subjected to in situ hybridization (ISH) for Epstein-Barr virus (EBV)-encoded RNA (EBER) according to the manufacturer's instructions.

\section{Results}

\section{Histopathologic and immunohistochemical findings}

The H\&E-stained sections of the inguinal lymph node biopsy specimen showed effacement of the nodal architecture by a diffuse infiltrate of small- to intermediate-sized cells with irregular nuclear contours, condensed chromatin, and a scant amount of pale to clear cytoplasm. The background displayed a prominent proliferation of high endothelial venules with arborization. Immunohistochemical analysis revealed that the cells were positive for $\mathrm{CD} 4, \mathrm{CD} 5, \mathrm{CD} 10$, and Bcl-6; weakly positive for $\mathrm{CD} 3$; and negative for CD79a, CD20, bcl-2, CD34, and CD30. The CD21 immunostain highlighted the expanded follicular dendritic meshwork. The Ki67 immunostain showed that the lymphoma cells had an intermediate proliferation rate. ISH for EBER was negative in the tumor cells. A diagnosis of AILT was established on the basis of lymph node features.

Staging bone marrow aspirates and trephine biopsy revealed a hypercellular $(85 \%$ cellular) marrow with approximately $40 \%$ involvement by AITL. A reticulin stain demonstrated grade 3 reticulin fibrosis with focal collagen fibrosis. The EBER stain by ISH was negative.

Peripheral blood showed leukocytosis with absolute lymphocytosis. Examination of the peripheral blood cells revealed numerous atypical lymphoid cells, which were small to intermediate in size with slightly irregular nuclear contours, condensed nuclear chromatin, and a moderate amount of pale cytoplasm (Fig. 1).

\section{Flow cytometry}

The second bone marrow biopsy and aspiration was performed a month after the lymph node biopsy. Flow cytometry of the bone marrow aspirate revealed an aberrant T-cell population $(5 \%)$ with an immunophenotype similar to that of the known lymphoma (Table 1). The flow cytometry assessment of peripheral blood cells collected around the same time revealed a clonal T-cell population (93\%) expressing CD45, CD2, CD4, bright CD5, CD7, and CD10, but not CD3 (Fig. 2). The lymph node biopsy was performed at the outside hospital and hence no flow data were available.

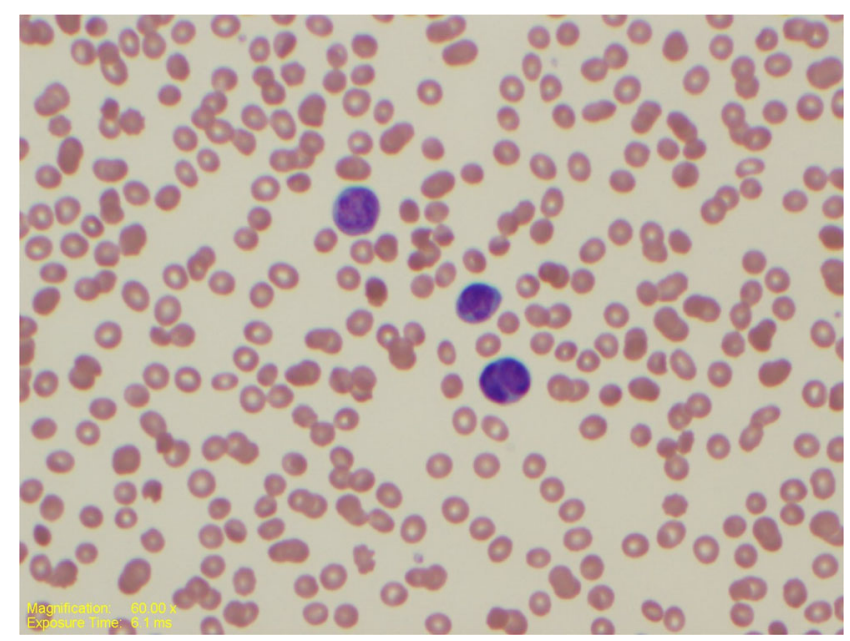

Fig. 1 Wright stain of a peripheral blood smear showing neoplastic lymphocytes (magnification $\times 40$ ) 
Table 1 Immunophenotypes of lymphoma cells in peripheral blood, lymph node, and bone marrow specimens

\begin{tabular}{llllll}
\hline & $\mathrm{CD} 3$ & $\mathrm{CD} 4$ & $\mathrm{CD} 5$ & $\mathrm{CD} 8$ & $\mathrm{CD} 10$ \\
\hline Peripheral blood & - & + & + & - & + \\
Lymph node & $+/-$ & + & + & - & + \\
Bone marrow & - & + & + & - & + \\
\hline
\end{tabular}

\section{Treatment and clinical course}

The patient underwent one course of high-dose hyper-CVAD. A repeat flow cytometry analysis of the peripheral blood cells after this treatment showed $93 \%$ lymphoma cells with an immunophenotypic profile identical to the primary AITL. Her disease did not respond to a second course of high-dose methotrexate/cytarabine, and she elected to pursue hospice care at home. The patient passed away from refractory disease a few days after discharge from the hospital.

\section{Discussion}

AITL is an aggressive peripheral T-cell lymphoma accounting for approximately 1-2\% of all non-Hodgkin lymphomas and around $20 \%$ of peripheral T-cell lymphomas. The lymph nodes are the primary site of involvement. In addition, spleen, liver, skin, and bone marrow are frequently involved [3, 4]. Despite treatment, the overall survival duration is poor and the prognosis remains dismal.

The presence of circulating lymphoma cells in AITL patients has been documented in a previous case series. In 1978, Pangalis et al. reviewed the peripheral blood and bone marrow biopsy specimens of 38 patients with the features of AITL and identified "immunocytes" in the peripheral blood smears of 12 of them. Because immunochemical analysis was not possible at that time, the cells could not be clearly identified nor the clinical correlations established [5]. More recently, in 2006,
Baseggio et al. examined the whole peripheral blood samples from six patients with AITL by flow cytometry and identified a fraction of $\mathrm{T}$ cells expressing CD10 (mean percentage $18.75 \%$, range 5-47 \%) [4]. Identification of CD10 expression in the neoplastic $\mathrm{T}$ cells by immunohistochemistry has also been described in extranodal dissemination of AITL. In a study by Attygale et al., aberrant CD10 expression was found in 10 cases of AITL with extranodal dissemination to cecum, tonsil, and lung [6]. Six cases had morphologic evidence of bone marrow involvement. However, only one of the six bone marrow specimens showed positive CD10 staining by immunohistochemistry. Those authors concluded that CD10 expression was maintained in the neoplastic $\mathrm{T}$ cells in the extranodal sites and hence early and accurate diagnosis of AITL can be achieved by immunohistochemical detection of CD10 expression.

Despite the description of circulating CD10-positive neoplastic T cells in AITL, no case with a prominent leukemic phase has been reported. In the case series published earlier, only $10-20 \%$ of the whole circulating lymphoid population was atypical [4]. Our patient presented with leukocytosis and absolute lymphocytosis, with $>90 \%$ of cells being neoplastic $\mathrm{T}$ cells. Given the history of lymphoma diagnosed in a lymph node, the presence of abnormal lymphocytes in the peripheral blood is strongly suggestive of lymphoma dissemination and must be followed up by careful examination of the circulating lymphocytes by morphologic and flow cytometric analyses. The present case suggests that a subset of cases of AITL or a certain variant is associated with a leukemic phase. This phase may be associated with an adverse prognosis. It is not known whether a certain type of treatment can cause a leukemic phase. In 1975, Lukes and Tindle described six cases of immunoblastic lymphadenopathy in which the disease symptoms appeared abruptly after administration of drugs such as penicillin, griseofulvin, or phenytoin [7].

Epstein-Barr virus has been suggested to play a role in the pathogenesis of AITL. Weiss et al., in a series of 23 cases of AITL, showed that EBV nucleic acid sequences were present
Fig. 2 Aberrant loss of CD3 (left) and co-expression of $\mathrm{CD} 5$ (right) in the CD4-positive tumor cell population
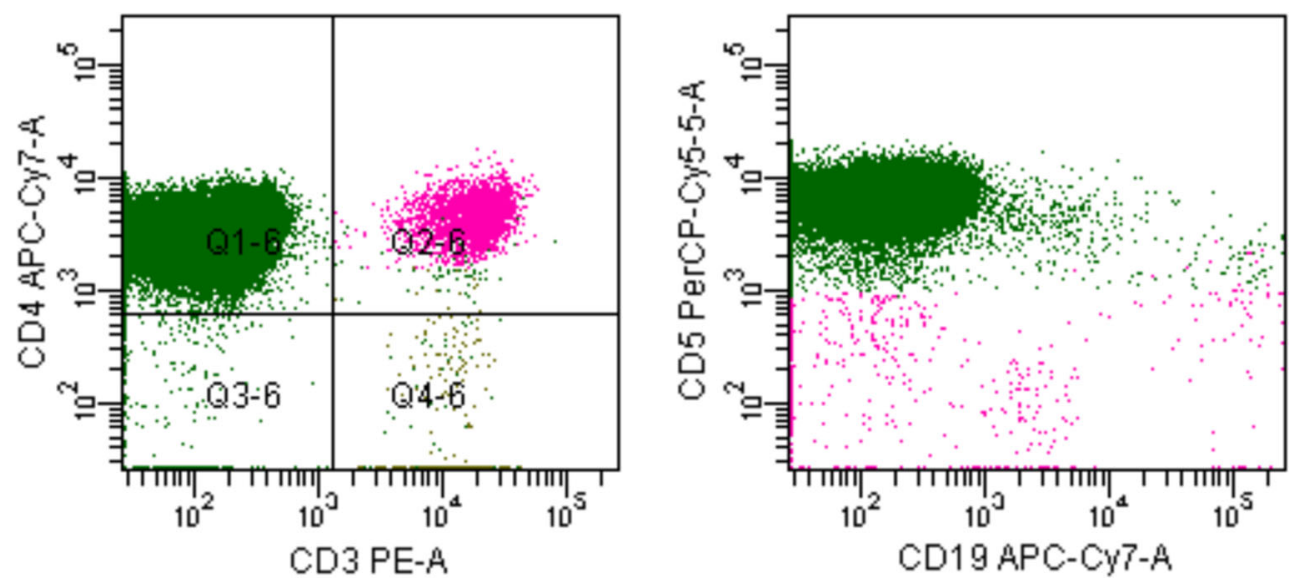
in B cells in $96 \%$ of the cases by either polymerase chain reaction (PCR) or ISH [8]. These proliferating B cells can occasionally progress to an EBV-positive B-cell lymphoma, either synchronous with or after the diagnosis of AITL [9]. In our case, however, the EBER staining was negative. Whether the leukemic phase represents an advanced phase of AITL with absence of the B cells or there is any correlation between EBV-negative status and a leukemic phase needs to be studied further.

\section{Compliance with ethical standards}

Conflicts of interest The authors declare that they have no conflicts of interest relevant to the manuscript submitted to Journal of Hematopathology.

\section{References}

1. Frizzera G, Moran EM, Rappaport H (1974) Angioimmunoblastic lymphadenopathy with dysproteinaemia. Lancet 1(7866):10701073
2. Nathwani BN, Rappaport H, Moran EM, et al. (1978) Malignant lymphoma arising in angioimmunoblastic lymphadenopathy. Cancer 41:578-606

3. Federico M, Rudiger T, Bellei M, et al. (2013) Clinicopathologic characteristics of angioimmunoblastic T-cell lymphoma: analysis of the international peripheral T-cell lymphoma project. J Clin Oncol $31: 240-246$

4. Baseggio L, Morel D, Delfau-Larue M-H, et al. (2006) Identification of circulating CD10 positive $\mathrm{T}$ cells in angioimmunoblastic T-cell lymphoma. Leukemia:296-303

5. Pangalis GA, Moran EM, Nathwani BN, et al. (1983) Angioimmunoblastic lymphadenopathy: long-term follow-up study. Cancer 52:318-321

6. Attygalle AD, Diss TC, Munson P, Isaacson PG, MQ D, Dogan A (2004) CD10 expression in extranodal dissemination of angioimmunoblastic T-cell lymphoma. Am J Surg Pathol 28(1):54 61

7. Lukes RJ, Tindle BH (1975) Immunoblastic lymphadenopathy. A hyperimmune entity resembling Hodgkin's disease. N Engl J Med 292(1): $1-8$

8. Weiss LM, Jaffe ES, Liu XF, Chen YY, Shibata D, Medeiros LJ (1992) Detection and localization of Epstein-Barr viral genomes in angioimmunoblastic lymphadenopathy and angioimmunoblastic lymphadenopathy-like lymphoma. Blood 79(7):1789-1795

9. Willenbrock K, Brauninger A, Hansmann ML (2007) Frequent occurrence of B-cell lymphomas in angioimmunoblastic T-cell lymphoma and proliferation of Epstein-Barr virus-infected cells in early cases. Br J Haematol 138:733-739 